\title{
QUALIDADE DO CAFÉ ARÁBICA POR DIFERENTES GRANULOMETRIAS
}

\author{
Wener Luis Soares'1, Tamara Machado Silva1', Kleso Silva Franco Junior², Giselle Prado Brigante² \\ ${ }^{1}$ Engenheiro Agronomo, CESEP- Centro Superior de Ensino e Pesquisa de Machado MG, Avenida Dr Athaide Pereira de Souza, S/N , \\ Machado MG 37750-000 \\ ${ }^{2}$ Professores Dr. do curso de agronomia do CESEP, Centro Superior de Ensino e Pesquisa de Machado MG, Avenida Dr Athaide \\ Pereira de Souza, S/N , Machado MG 37750-000 \\ Autor para correspondência: Kleso Silva Franco Junior, kleso.junior@yahoo.com.br
}

\begin{abstract}
RESUMO: 0 café há séculos é uma das bebidas mais apreciadas do mundo. Tendo sua origem nas regiões árabes, e com o passar do tempo difundiu-se pela América do Sul e África. O Brasil possui solo e clima propícios para o cultivo do café. $O$ presente trabalho apresenta uma análise sobre a qualidade da bebida do café da espécie Coffea arabica obtidas por diferentes tipos de granulometria (peneiras), onde foram realizados experimentos com diferentes amostras de café colhidas de lavouras cultivadas em propriedades situadas no município de Campos Gerais, MG. A colheita foi realizada por derriça manual no pano, para a classificação foram utilizadas peneiras de números 19 a 10 (para grãos chatos) e 13 a 8 (para grãos moca), na seguinte ordem: Peneira 19 chato; Peneira 13 moca; Peneira 18 chato; Peneira 12 moca; Peneira 17 chato; Peneira 11 moca; Peneira 16 chato; Peneira 10 moca; Peneira 15 chato; Peneira 9 moca; Peneira 14 chato; Peneira 13 chato; Peneira 8 moca; Peneira 10 chato; Fundo plano. Após a classificação, os cafés foram agrupados da seguinte forma: Chato graúdo: peneiras 19, 18 e 17, Chato médio: peneiras 16 e 15, e Chato miúdo: peneira 14 e menores, Moca graúdo: peneiras 13, 12 e 11 e Moca miúdo: peneiras 10 e inferiores. Aplicou-se análise sensorial para tamanho do grão, procedência do café, torrefação entre outros fatores. Observou-se que os tratamentos ao qual utilizaram peneira miúda e moca miúda obtiveram resultados com melhores notas na tabela SCAA, sendo superiores em relação as demais.
\end{abstract}

\section{PALAVRAS CHAVES: Produção de café, Classificação sensorial, Bebida.}

\section{QUALITY OF ARABIC COFFEE FOR DIFFERENT GRANULOMETRIES}

ABSTRACT: Coffee for centuries is one of the most appreciated drinks in the world. Having its origin in the Arab regions, and with the passage of the time spread by South America and Africa. Brazil has favorable soil and climate for coffee cultivation. The present work presents an analysis on the coffee quality obtained by different types of granulometry (sieves), where experiments were carried out with different samples of coffee harvested from crops grown in properties located in the city of Campos Gerais, MG. Harvesting was carried out by manual melting on the cloth. Sieves of numbers 19 to 10 (for flat grains) and 13 to 8 (for mocha grains) were used in the following order: Sieve 19 flat; Sieve 13 mocha; 18 flat screen; Sieve 12 mocha; Sieve 17 flat; Sieve 11 mocha; Sieve 16 boring; Sieve 10 mocha; Sieve 15 boring; Sieve 9 mocha; Sieve 14 flat; Sieve 13 boring; Sieve 8 mocha; Sieve 10 boring; Flat background. After grading, the coffees were grouped as follows: Large dull: sieves 19, 18 and 17, Medium flat: 16 and 15 sieves, and Kidnapping: 14 and smaller sieves, Large mocha: sieves 13, 12 and 11 and Mocha sieves: 10 and lower sieves. Sensory analysis was applied for grain size, coffee origin, roasting among other factors. It was observed that the treatments that used small sieve and small moca obtained results with better scores in the SCAA table, being superior in relation to the others.

KEYWORDS: Coffee production, Sensory classification, Beverage.

\section{INTRODUÇÃO}

$O$ café é uma das bebidas mais consumidas no mundo, sendo o Brasil o maior produtor desta matéria prima, com uma produção na última safra de 2017/2018 de aproximadamente 61,7 milhões de sacas (CONAB,
2018), o café tem assim, um papel de destaque na economia brasileira.

Há maior parte da produção brasileira é exportada para diversos países, nos quais estão cada vez mais exigentes em relação a qualidades dos grãos, 
pois como em qualquer outra bebida, o consumidor quer ter prazer em degusta-la, estando assim, disposto a pagar mais por um café de melhor qualidade. Visando atender estes mercados os produtores brasileiros começaram a se preocupar em produzir cafés com qualidade superior.

Diversos fatores interferem na qualidade dos grãos, entre eles estão: genética da planta, ataques de pragas e doenças, manejo errado no pós colheita, intenpéries climáticas e o processo de torração no qual os grãos sofrem algumas reações físicas e químicas importantes, para evidenciar as características de sabor e 0 aroma.

Outro processo importante é o de beneficiamento do grão, onde se nota que os produtores não se preocupam em separar os grãos pelos seus tamanhos, pois esta separação padronizará o lote, dando a eles uma torra mais uniforme impedindo que grãos queimem, evitando um sabor desagradável no produto.

Após 0 beneficiamento 0 café passa por diversos tipos de classificações onde irá determinar os seus aspectos físico e sensoriais, nos quais gerarão 0 preço final do produto, ainda há poucos estudos sobre a influência da seleção por granulometria do grão influencia nestes processos.

Considerando estes aspectos percebe-se a importância de se realizar trabalhos, visando analisar se há interferências da granulometria do grão na qualidade final da bebida do café.

\section{MATERIAL E MÉTODOS}

Para a realização da pesquisa, foi instalado um experimento utilizando três amostras de café da espécie Coffea arabica colhidas de lavouras cultivadas nas propriedades rurais, sitio Três Barras e do Sitio Toca que estão situadas no município de Campos Gerais, MG, ambas as propriedades estão localizadas em altitude média de $800 \mathrm{~m}$ e com as respectivas coordenadas geográficas: latitude $21^{\circ} 13^{\prime} 47^{\prime \prime} \mathrm{S}$ e $21^{\circ}$ $13^{\prime} 45^{\prime \prime}$ S e longitude de $45^{\circ} 54^{\prime} 15^{\prime \prime} \mathrm{O}$ e $45^{\circ} 54^{\prime} 07^{\prime \prime} \mathrm{O}$.

Duas amostras foram colhidas no sitio Três Barras sendo uma da cultivar Mundo novo (vermelho) e a outra da cultivar Catuai (vermelho) ambas com dez anos de idade aproximadamente. A outra amostra foi de uma cultivar Mundo Novo (vermelho), com aproximadamente doze anos de idade, colhida no Sitio Toca.
Os frutos foram colhidos por derriça manual no pano, quando $80 \%$ dos frutos atingiram a maturidade fisiológica, foram secos com boas práticas de manejo em terreiros de concreto nas respectivas propriedades, até atingir teores de água entre 11 a 12\% (b.u). Depois da secagem os cafés foram beneficiados por maquinas (ambulante no sitio Três Barras e estacionaria no sitio Toca), e acondicionados em sacos de estopa e enviados para armazenamento.

Foi retirada uma amostragem de cada saco de estopa dos respectivos lotes, e posteriormente foram classificados os grãos pelo seu tamanho, utilizado peneiras intercaladas, para os grãos chatos as de números 19 a 10 e de grãos mocas de números 13 a 8 na seguinte ordem: Peneira 19 chato; Peneira 13 moca; Peneira 18 chato; Peneira 12 moca; Peneira 17 chato; Peneira 11 moca; Peneira 16 chato; Peneira 10 moca; Peneira 15 chato; Peneira 9 moca; Peneira 14 chato; Peneira 13 chato; Peneira 8 moca; Peneira 10 chato; Fundo plano. Após a classificação, os cafés foram agrupados da seguinte forma: Chato graúdo: peneiras 19, 18 e 17, Chato médio: peneiras 16 e 15, e Chato miúdo: peneira 14 e menores, Moca graúdo: peneiras 13, 12 e 11 e Moca miúdo: peneiras 10 e inferiores. Posteriormente, foram acondicionadas em sacos plásticos e armazenadas em temperatura ambiente, até a realização da análise sensorial.

Cada amostra foi composta por $350 \mathrm{~g}$ de café beneficiados, onde foram retirados os defeitos intrínsecos e extrínsecos, classificando assim quanto ao seu tipo, (de acordo com a Instrução Normativa N8 (BRASIL, 2003), foi medida a umidade de cada amostra, e posteriormente a amostra pronta, foi torrada e submetida a uma análise sensorial sendo pontuada a amostra de acordo com a metodologia da Associação Americana de Cafés Especiais (SCAA, 2008), as provas sensoriais dos cafés foram realizadas por tres provadores capacitados e que estavam em plena a atividade.

0 delineamento estatístico utilizado foi 0 inteiramente casualizado, tendo como tratamentos: o tamanho do grão, sendo separados em 6 níveis (grão chato: peneira graúda, media, miúda; grão moca: peneira graúda e miúda e a testemunha a amostra sem a separação, bica corrida), e a procedência do café 3 níveis (amostras de diferentes cafés e locais) com 2 repetições (provadores) assim teremos $6 \times 3$ $x 2$ totalizando 36 parcelas. Os dados obtidos serão 
analisados pelo software Sisvar 4.0 (Ferreira, 2014), e as médias foram comparadas pelo teste de Scott Knott, a $5 \%$ de probabilidade.

\section{RESULTADOS E DISCUSSÃO}

Os resultados obtidos foram submetidos a análise estatística e seguem na tabela 1 , sendo em relação ao parâmetro qualidade, pontuação na metodologa SCAA. Os tratamentos peneira miúda e moca miúda foram estatisticamente superiores em relação aos demais, corroborando pelo que já foi comprovado por Reis et al. (2013), onde, estes, demonstraram que os grãos de menores granulometria apresentaram notas superiores no atributo final quando processados de forma natural.

Tabela 1. Classificação das amostras de café de acordo com a sua granulométrica

\begin{tabular}{lccc}
\hline \multicolumn{1}{c}{ Tratamentos } & Pontuação SCAA & Quantidade de defeitos & Teor de umidade \\
\hline Bica corrida & $75,4 \mathrm{~B}$ & $95,66 \mathrm{~B}$ & $11,73 \mathrm{~A}$ \\
Peneira graúda & $72,9 \mathrm{~B}$ & $39,66 \mathrm{C}$ & $11,7 \mathrm{~A}$ \\
Peneira media & $73,7 \mathrm{~B}$ & $62,33 \mathrm{C}$ & $11,7 \mathrm{~A}$ \\
Peneira miúda & $78,03 \mathrm{~A}$ & $324,66 \mathrm{~A}$ & $11,63 \mathrm{~A}$ \\
Moca graúda & $54,30 \mathrm{C}$ & $86,002 \mathrm{~B}$ & $11,6 \mathrm{~A}$ \\
Moca miúda & $76,13 \mathrm{~A}$ & $84,00 \mathrm{~B}$ & $11,6 \mathrm{~A}$ \\
CV \% & 22,50 & 17,3 & 31,2 \\
\hline
\end{tabular}

*Médias seguidas pela mesma letra e números diferentes diferem estatisticamente. Scott-Knott, a $5 \%$ probabilidade.

No atributo quantidade de defeitos, os que apresentaram uma menor quantidade de defeitos foram os tratamentos peneira graúda e peneira media, diferindo estatisticamente das demais. Os tratamentos moca miúda, graúda e bica corrida foram estatisticamente superiores ao tratamento peneira miúda, tal fato pode ser explicado, pois os grãos quebrados passam pelas peneiras de maior crivo, se acumulado nas menores. Em relação a umidade não houve diferença significativa entres os tratamentos.

Em relação ao tipo de café (Da Fonseca et al., 2007; Rocha et al., 2009; Carvalho et al., 2011) que apresentam as espécies Coffea arabica e Coffea canephora, entretanto, chamam a atenção para a classificação dos grãos quanto ao tamanho e a cor, pois segundo estes autores, o fato de não manter os grãos do mesmo tamanho poderá prejudicar a classificação, uma vez que os grãos menores possuem tempo de torrefação menor que os demais grãos, o que poderia trazer amargor para a bebida.

Rosseti (2007) e Giomo; Borém (2011) concordam com os autores já citados acrescentando que 0 aroma, a cor, quantidade de defeitos influencia diretamente na qualidade da bebida, que poderá alterar se não for armazenada em local apropriado.

Tomaz (2011) e Giomo; Borém (2011) corroboram da mesma ideia, afirmando que essa classificação deverá ser realizada no primeiro estágio pós colheita, ou seja, no processo de secagem.
Malta (2011), Tomaz (2011), Giomo; Borém (2011) e Carvalho et al. (2011), afirmam que a temperatura de infusão, a água e o tempo de infusão poderão modificar o aroma e o sabor, por isso as amostras devem sempre permanecer em recipiente hermético, adicionando a água somente no momento da degustação.

Concluo que os tratamentos peneira miúda e moca miúda, obtiveram melhores notas na tabela SCAA, sendo superiores em relação as demais.

Em relação a classificação pelo seu tipo as amostras que obtiveram menores números de defeitos foram às peneiras graúdas e medias classificando as suas medias respectivamente como tipo 4-39 defeitos e tipo 5-62 defeitos.

\section{AGRADECIMENTOS}

Ao CESEP - Centro Superior de Ensino e Pesquisa de Machado MG pelo apoio na realização deste e a Revista Revista Ciência Agricola pela oportunidade de estar proporcionando a difusão destes resultados que poderão ser uteis aos cafeicultores.

\section{REFERÊNCIAS BIBLIOGRAFICAS}

BRASIL. Ministério da Agricultura, Pecuária e Abastecimento. Instrução Normativa $n^{0} 16$, de 24 de maio de 2010. Estabelece o Regulamento Técnico para o Café Torrado em Grão e Café Torrado e Moído. 
Diário Oficial da União, Brasília, 25 maio 2010. Seção 1, p.11. Disponível em: <http://www.abic.com.br/ publique/media/CONS_leg_regulamentotecnicolN16. pdf> Acesso em: 10 abr. 2017.

BRASIL. Ministério da Agricultura, Pecuária e Abastecimento. Instrução Normativa $n^{0} 8$, de 11 de junho de 2003. Diário Oficial da União, Poder Executivo, Brasília, 13 jun. 2003. Seção 1, p. 8. Disponível em: <http://www.abic.com.br/publique/media/CONS_leg_ instnormativa0803.pdf>. Acesso em: 10 abr. 2017.

Borem, F. M.; Coradi, P. C.; Saath, R.; Oliveira, J. A. Qualidade do café natural e despolpado após secagem em terreiros e com altas temperaturas. Ciências Agrotecnologia, 2008, 32, 5, 1609-1615. Disponível em: <https://repositorio.unesp.br/bitstream/ handle/11449/30177/S141370542008000500038. pdf?sequence $=1$ \&isAllowed=y>. Acesso em 13 de mar.2017

Carvalho, G.R.; Rezende, J. C. de ; Botelho, C.E.; Ferreira, A. D.; Pereira, A. A.; Oliveira, A. C. B. Melhoramento genético do café visando à qualidade de bebida, Informe Agropecuário, 2011, 32, 261, 30-38. Disponível em: <http://www.sapc.embrapa.br/arquivos/ consorcio/informe_agropecuario/ia_cafe_producao_ qualidade.pdf>. Acesso em: 13 de abr 2017

Carvalho, V. D.; Chagas, S. J. R.; Souza, S. M. C. Fatores que afetam a qualidade do café. Informe Agropecuário, 1997, 18, 187, 5-20.

CONAB. Acompanhamento da safra Brasileira de café, 2018, 5, safra 2018, 4, 20-25.

Da Fonseca, A. F. A.; Ferrão, R. G.; Verdin Filho, A.C.; Volpi, P.S. Qualidade do café conilon: operações de colheita e pós-colheita. In: DA FONSECA, A. F. A. Café Conilon. Vitória ES: Incaper, 2007, 501-520.

Ferreira, D. F. Sisvar: um guia dos seus procedimentos de comparações múltiplas Bootstrap. Ciência e Agrotecnologia, 2014, 38, 2, 109-112.

Giomo, G. S.; Borém, F. M. Cafés especiais no Brasil: opção pela qualidade. Informe Agropecuário, 2011, 32, 261, 7-16. Disponível em:<http://www.sapc.embrapa. br/arquivos/consorcio/informe_agropecuario/ ia_cafe producao_qualidade.pdf>. Acesso em: 15 de abr 2017.
Laviola, B. G.; Mauri, A. L.; Martinez, H. E. P.; Araújo, E. F.; Neves, Y. P. Influência da adubação na formação de grãos mocas e no tamanho de grãos de café (Coffeaarabica L.). Coffee Science, 2006, 1, 1, 39-42.

Lopes, L. M. V. Avaliação da qualidade de grãos crus e torrados de cultivares de cafeeiro (Coffe arábica L.). Dissertação de Mestrado em Ciência dos Alimentos, Universidade Federal de Lavras, UFLA, Lavras, 95p, 2000.

Malta, M. R. Critérios utilizados na avaliação da qualidade do café. Informe Agropecuário, 2011, 32, 261, 126. Disponível em:<http://www.sapc.embrapa. br/arquivos/consorcio/informe_agropecuario/ ia_cafe producao_qualidade.pdf $>$. Acesso em: 13 de abr 2017.

Malta, M. R.; Pereira, R. G. F. A; Chagas, S. J. R. Condutividade elétrica e lixiviação de potássio do exsudato de grãos de café: alguns fatores que podem influenciar essas avaliações, Revista Ciência Agrotecnologia, 2005, 29, 1015 a 1020p;5, .

Marcomini, G. R. Aspectos econômicos financeiro da produção de café convencional e de café espacial. Revista Científica da FHO| UNIARARAS, 2013, 1, 1-11. Disponível em:<http://www.uniararas.br/ revistacientifica/_documentos/art. 2 -007-2011.pdf>. Acesso em: 13 de abr 2017.

Martinez, H. E. P.; Tomaz, M. A.; Sakiyama, N.S. Guia de Acompanhamento das Aulas de cafeicultura. $2^{\mathrm{a}}$ ed. Viçosa: UFV, 2007152 p.

Mendonça, L. M. V. L.; Pereira, R. G. F. A.; Borém, F. M.; Almeida, S. R. de.; Garcia, A. W. R..; Mendonça, J. M. A. Classificação por peneira de grãos de Coffea arabica L. avaliada por meio de análise multivariada, Trabalho apresentado no Simpósio de Pesquisa dos Cafés do Brasil (4. : 2005 : Londrina, PR). Anais. Brasília, D.F. : Embrapa Café, 2005.

Fundação PROCAFÉ, Varginha, [S.I.], [2005], Disponível em:<http://www.sapc.embrapa. br/arquivos/ consorcio/spcb_anais/simposio4/p249.pdf>. Acesso em: 09 abr. 2017.

Paiva, E. F. F. Análise sensoriais dos cafés especiais de Minas Gerais. Dissertação de Mestrado (Ciências dos 
Alimentos); Universidade Federal de Lavras; UFLA, Lavras; 55 p, 2005. Disponível em: <http:/l repositorio.ufla.br/jspui/bitstream/1/2843/1/ DISSERTA\%C3\%87\%C3\%830_An\%C3\%A1lise \%20 sensorial\%20dos\%20caf\%C3\%A9s\%20especiais\%20 do $\% 20$ estado $\% 20$ de $\% 20$ Minas $\% 20$ Gerais.pdf>. Acesso em: 15 abr. 2017.

Reis, T. H. P.; Guimarães, P. T. G, Furtini Neto, A. E, Gerra, A. F., Curi, N.; Soil phosphorus dynamics and availability and irrigated coffee yeld. Revista Brasileira de Ciências do Solo, 2011, 35, 503-512.

Rocha, R. B.; Teixeira, A. L., Ramalho, A. R., Souza, F. F., Melhoramento de Coffeacanephora: Consideraçõese Metodologia. [S.I.]: Embrapa, 2009. 128p. Disponívelem: <https://www.alice.cnptia.embrapa.br/alice/bitstream/ doc/1040710/1/CafenaAmazoniaRodrigoRocha. pdf>. Acesso em:09 abr. 2017.

Rossetti, R. P. Determinação de fenóis totais em frutos do café: Avaliações em diferentes fases de maturação. 2007. 72f. Dissertação (Mestrado em Ciências) Universidade de São Paulo. São Carlos.
SCAA. Specialty Coffee Association Of America. Protocols - CuppingSpecialtyCoffee. Long Beach: SCAA. 2009. 7p, Disponivel em: <http://www.scaa. org/?page=resources\&d=cupping-protocols $>$, Acesso em: 01 maio 2017.

SCAA. Specialty Coffee Association Of America. Protocolo para Análise Sensorial de Café- Metodologia SCAA. Doc. V- Português e. Rev. Dez 2008. 13p.

Tomaz, M. A. Guia de acompanhamento das aulas de cafeicultura, 2011,60p. Universidade Federal do Espirito Santo; Centro de Ciências Agrarias, Departamento de Produção Vegetal, Alegre ES, 2011, Disponível em: <file:///C:/Users/Windows/Downloads/Apostila\%20 Cafeicultura.pdf>. Acesso em: 01 maio 2017.

Uejo Neto, E. Compreendendo os cafés especiais. RevistaAttalea Agronegócios, 2008,2ped 26. Disponível em: <https://issuu.com/revistadeagronegocios/docs/ name7f4594/22>. Acesso em: 01 maio 2017. 
